\title{
Effects of regular-moderate exercise on high-fat diet-induced intramyocellular lipid accumulation in the soleus muscle of Sprague-Dawley rats
}

\author{
Kyung-Wan Baek ${ }^{1,2}$, Hee-Jae Cha², Mee Sun Ock², Hong Soo Kim³ ${ }^{3}$, Jeong-An Gim", Jung-Jun Park ${ }^{1, *}$ \\ 'Division of Sport Science, Pusan National University, Busan, Korea \\ 2Department of Parasitology and Genetics, Kosin University College of Medicine, Busan, Korea \\ ${ }^{3}$ Department of Gastroenterology/Hepatology, Soonchunhyang University College of Medicine, Cheonan, Korea \\ ${ }^{4}$ Department of Biological Sciences, College of Natural Sciences, Pusan National University, Busan, Korea
}

Previously, we monitored the expression level of the pro-apoptotic proteins caspase- 3 and cleaved poly-ADP-ribose polymerase in the skeletal muscle of high-fat diet-induced obese rats in order to assess muscle damage. In this study, we analyzed whether exercise or dietary adjustment was more effective at preventing high-fat diet-induced muscle damage. High-fat diet-induced obese rats were divided into three groups: the high-fat diet (HFD), the combined high-fat diet and exercise (HFD+EXE), and the dietary adjustment (DA) groups. For 6 weeks, the HFD+EXE group was subjected to exercise on an animal treadmill. Capsase-3 protein was quantified, and histopathology of the soleus muscle was performed. Both the HFD+EXE and DA interventions resulted in a reduction of lipid accumulation in the soleus muscle, and nucle- us infiltration was significantly lower in the DA group. The inflammatory response, caspase-3 level, and relative muscle weight were significantly higher in the HFD+EXE group compared to the HFD group. An increase in intramyocellular lipids in the soleus muscle by obesity and exercise stimulated apoptosis. When the rats exercised, muscle growth was normal and unrelated to the effects of lipid accumulation. These data indicate that exercise was more effective than dietary adjustment in reducing lipid accumulation and increasing muscle metabolism.

Keywords: Regular-moderate exercise, High-fat diet, Obesity, Skeletal muscle, Lipid accumulation, Cell damage

\section{INTRODUCTION}

Recently, the prevalence of obesity and associated metabolic complications has increased. Obesity and lipid accumulation in the body are strongly related to type 2 diabetes mellitus (T2DM) and other conditions. Furthermore, obesity can be a factor in cellular dysfunction and cell death (e.g., lipotoxicity) (Kusminski et al., 2009). The main cause of obesity is an energy imbalance between the intake of excess calories, often from a high-fat diet, and the expenditure of calories. Specifically, consumption of a high-fat diet leads to resistance to leptin and insulin (Dube et al., 2007; Van Heek et al., 1997) and accumulation of intramyocellular lipids (IMCLs) (Bastie et al., 2004; Hegarty et al., 2002; Phillips et al., 1996). Because excessive intracellular accumulation of IMCLs is related to increased insulin resistance, it can also induce mitochondrial dysfunction (Schrauwen, 2007; Schrauwen-Hinderling et al., 2005; Schrauwen-Hinderling et al., 2006).

In fact, obesity correlates with increased IMCL, and people with low levels of physical activity have a chronic positive energy balance that can lead to lipid accumulation in the skeletal muscle (Dubé et al., 2011; Mikines et al., 1988). In addition, intramuscular IMCL accumulation is associated with pro-apoptotic signaling and dysregulated lipid metabolism (Baek et al., 2013; Dubé et al., 2011; Siu et al., 2004). Our previous study confirmed that a high-fat diet dramatically elevated IMCL levels and pro-apoptotic protein expression in the skeletal muscles of rats. Moreover,
${ }^{\star}$ Corresponding author: Jung-Jun Park (D) https://orcid.org/0000-0002-2518-7225 Division of Sport Science, Pusan National University, 2 Busandaehak-ro 63beon-gil, Geumjeong-gu, Busan 46241, Korea

Tel: +82-51-510-2713, Fax: +82-51-510-3746, E-mail: jjparkpnu@pusan.ac.kr Received: November 30, 2017 / Accepted: January 16, 2018
This is an Open Access article distributed under the terms of the Creative Commons Attribution Non-Commercial License (http://creativecommons.org/licenses/by-nc/4.0/) which permits unrestricted non-commercial use, distribution, and reproduction in any medium, provided the original work is properly cited. 
relative muscle weight (muscle weight/body weight) decreased in the high-fat diet group (Baek et al., 2013). Taken together, these results suggest that lipid accumulation in muscle cells increases the degree of abnormality and apoptosis. However, exercising not only decreases the overall weight and proportion of body fat, but also plays a role increasing insulin sensitivity and muscle metabolism (Borghouts and Keizer, 2000; Bradley et al., 2008; Devlin, 1992; Goodyear and Kahn, 1998). However, prolonged exercise can cause skeletal muscle damage and apoptosis (Carraro and Franceschi, 1997; Phaneuf and Leeuwenburgh, 2001). On the basis of these considerations, the current study aimed to determine whether exercise or dietary adjustment is more effective at preventing high-fat diet-induced muscle damage.

In this study, we examined the changes in caspase-3 (pro-apoptotic protein) in muscle cells through exercise and diet for 6 weeks in high-fat diet-induced obese Sprague-Dawley rats.

\section{MATERIALS AND METHODS}

\section{Animals}

Three-week-old male Sprague-Dawley rats $(\mathrm{n}=21)$ were purchased from Central Laboratory Animal (Seoul, Korea). The rats were kept in a room retained at $22^{\circ} \mathrm{C}-24^{\circ} \mathrm{C}$ with $50 \%-60 \%$ relative humidity and a 12-hr light/12-hr dark cycle. All animals were fed a high-fat diet containing 45\% fat (D12451, Research Diets, New Brunswick, NJ, USA) for 6 weeks. After 6 weeks, the high-fat diet-induced obese animals were divided randomly into three groups: the high-fat diet group (HFD), the combined highfat diet and exercise group (HFD+EXE), and the dietary adjustment group (DA). At that point, the HFD and HFD+EXE groups maintained a high-fat diet, and the DA group switched from a high-fat diet to a normal diet. All mice were provided ad libitum access to both feed and water.

\section{Exercise protocol}

The HFD+EXE group was subjected to exercise on an animal treadmill (DJ-344, Daejong Instrument Industry, Seoul, Korea). The exercise consisted of a 6-week accommodation phase with increasing exercise intensity (weeks $1-3,15 \mathrm{~m} / \mathrm{min}$ for 30,45 , or 60 min, respectively; weeks $4-5,20 \mathrm{~m} / \mathrm{min}$ for 30 or $45 \mathrm{~min}$, respectively), followed by a 1-week consistent training period $(20 \mathrm{~m} /$ $\mathrm{min}$ for $60 \mathrm{~min}$ ). Training was conducted 5 times a week between the hours of 6:00 a.m. and 7:00 p.m.. As a control, animals in the other groups were placed on an idle treadmill for the same time period. Before each training session, all running rats were allowed a 5-min warm-up phase with slowly increasing speed (Boor et al., 2009). Animal studies were approved by the Institutional Animal Care and Use and Committee of Pusan National University (approval number: PNU-2010-000106).

\section{Measurements}

\section{Body, muscle, and fat weights}

The body weight and food intake of each animal were measured weekly throughout the study. Following a 10-hr overnight fast, all animals were sacrificed $48 \mathrm{hr}$ after the last training session under ethyl ether general anesthesia. The soleus muscle was removed, washed in $0.9 \%$ cold saline, and immediately placed in liquid nitrogen. A portion of muscle tissue was immediately frozen in liquid nitrogen. All muscle samples were stored at $-80^{\circ} \mathrm{C}$ until further analysis. Abdominal adipose tissue and soleus muscle weights were measured using a microanalytical scale (AdventurerTM, Ohaus, Parsippany, NJ, USA).

\section{Histological analysis}

Hematoxylin and eosin $(\mathrm{H} \& \mathrm{E})$ staining: For $\mathrm{H} \& \mathrm{E}$ staining, sections were deparaffinized, hydrated, stained with hematoxylin for $5 \mathrm{~min}$. The sections were rinsed with water for $10 \mathrm{~min}$. The sections were then decolorized using $1 \% \mathrm{HCl}$, rinsed with water for $10 \mathrm{~min}$, stained with eosin for $1 \mathrm{~min}$, and dehydrated in gradient alcohols. Then, the sections were cleared in xylene prior to being sealed with neutral gum. $\mathrm{H} \& \mathrm{E}$ staining was used to evaluate macrophage infiltration, inflammation response, and cell regeneration.

\section{Oil Red O staining}

Frozen sections $(4 \mu \mathrm{m})$ from biopsy samples were placed on uncoated glass slides to determine the IMCL content in the soleus muscle using Oil Red O (ORO) staining.

\section{Casapase-3 Western blot}

Western blot analysis was performed to detect caspase- 3 expression in the muscle tissue. Soleus muscle tissue was homogenized in protein extraction solution (PRO-PREP, iNtRON Biotechnology, Seongnam, Korea). The homogenate was centrifuged at 13,000 rpm for $5 \mathrm{~min}$ at $4^{\circ} \mathrm{C}$, and the supernatant was collected. The protein concentration was determined using a Bradford protein assay. Twenty micrograms of soluble protein were boiled for 5 min at $100^{\circ} \mathrm{C}$ in Laemmli buffer, loaded onto a $12 \%$ polyacrylamide gel, and electrophoresed for $1.5 \mathrm{hr}$ at $20^{\circ} \mathrm{C}$. The gel was blotted onto a nitrocellulose membrane (Bio-Rad, Hercules, CA, 
USA) and stained with Ponceau S (Sigma-Aldrich, St Louis, MO, USA) to confirm equal loading and transfer of proteins from each lane to the membrane. The membrane was blocked in 5\% skim milk in Tris-buffered saline with $0.05 \%$ Tween 20 at room temperature for $1 \mathrm{hr}$ and probed with rabbit anti-caspase- 3 polyclonal antibody (1:1,000, dilution, \#9662, Cell Signaling Technology, Beverly, MA, USA) overnight at $4^{\circ} \mathrm{C}$. The membrane was incubated in anti-rabbit IgG secondary antibody (Abcam, Cambridge, $\mathrm{UK}$ ), and the signals were developed using a chemiluminescence reagent. The results were quantitated using the FluorChem HD2 imaging system (Alpha Innotech, San Leandro, CA, USA).

\section{Statistical analysis}

Statistical analysis was performed using the IBM SPSS ver. 18.0 (IBM Co., Armonk, NY, USA). One-way analysis of variance was used to examine the differences among the groups, and the Duncan test was used for post boc analysis. The results were considered statistically significant at $P<0.05$.

\section{RESULTS}

\section{Body weight gain, food intake, and food efficiency ratio}

The initial body weight was similar among the three groups. To evaluate the effects of the exercise intervention and the dietary adjustment, total weight gain and the food efficiency ratio (FER) were measured for each animal (Table 1). The total weight gain was significantly $(P<0.05)$ different among the groups $(\mathrm{HFD}>\mathrm{DA}>\mathrm{HFD}+\mathrm{EXE})$. Also, the FER was significantly $(P<0.05)$ higher in the high-fat diet groups $(\mathrm{HFD}>\mathrm{HFD}+\mathrm{EXE}$ $>\mathrm{DA})$.

\section{Abdominal adipose tissue weight and relative muscle weight}

As expected, the abdominal adipose tissue weight was signifi- cantly $(P<0.05)$ higher in the high-fat diet group compared to both the exercise and dietary adjustment groups (Fig. 1A). The relative muscle weight of the HFD+EXE group (Fig. 1B) was significantly higher than that of the HFD group $(P<0.05)$, but the DA group was not significantly different from the other groups.


Fig. 1. A high-fat diet led to increased abdominal adipose tissue weight, and exercise led to increased relative soleus muscle weight. (A) Increased abdominal adipose tissue means that obesity was induced. (B) The increase in relative muscle weight demonstrates that exercise increased pure muscle mass (excluding fat). HFD, high-fat diet; HFD+EXE, combined high-fat diet and exercise; DA, dietary adjustment.

Table 1. Body weight gain, food intake, and food efficiency ratio (FER) in high-fat diet-induced obese rats treated with exercise and diet adjustment

\begin{tabular}{|c|c|c|c|c|c|}
\hline Measure & $\operatorname{HFD}(A, n=7)$ & $\mathrm{HFD}+\operatorname{EXE}(\mathrm{B}, \mathrm{n}=7)$ & $\mathrm{DA}(\mathrm{C}, \mathrm{n}=7)$ & $F$ & Posthoc \\
\hline Initial weight (g) & $486.74 \pm 14.13$ & $486.45 \pm 10.40$ & $486.3 \pm 14.82$ & 0.00 & $N S^{b)}$ \\
\hline Final weight (g) & $650.79 \pm 24.41$ & $537.7 \pm 24.75$ & $608.14 \pm 21.62$ & $40.83^{* * *}$ & $\mathrm{~B}<\mathrm{C}<\mathrm{A}$ \\
\hline Total weight gain (g) & $164.04 \pm 25.09$ & $51.25 \pm 19.61$ & $121.86 \pm 13.16$ & $4.56^{*}$ & $\mathrm{~B}<\mathrm{C}<\mathrm{A}$ \\
\hline Average weight gain (g/wk) & $27.34 \pm 2.34$ & $8.54 \pm 2.10$ & $20.30 \pm 2.19$ & $57.4^{* * *}$ & $\mathrm{~B}<\mathrm{C}<\mathrm{A}$ \\
\hline Food intake (g/wk) & $117.35 \pm 9.95$ & $92.59 \pm 5.25$ & $175.53 \pm 6.63$ & $219.29 * * *$ & $\mathrm{~B}<\mathrm{A}<\mathrm{C}$ \\
\hline FER ${ }^{c \mid}$ & $0.23 \pm 0.03$ & $0.09 \pm 0.03$ & $0.01 \pm 0.01$ & $48.71^{* * *}$ & $\mathrm{C}<\mathrm{B}<\mathrm{A}$ \\
\hline
\end{tabular}

Values are presented as mean \pm standard deviation. Rats were divided into three groups: high-fat diet (HFD), combined high-fat diet and exercise (HFD+EXE), and dietary adjustment (DA) groups.

alThe data were analyzed using the Duncan test. ${ }^{\mathrm{b}}$ No stastically significant difference among group. ${ }^{\mathrm{c}} \mathrm{FER}=$ body weight gain $(\mathrm{g} / \mathrm{wk}) / \mathrm{food}$ intake $(\mathrm{g} / \mathrm{wk}) .{ }^{*} P<0.05$. ${ }^{* * *} P<0.001$. 


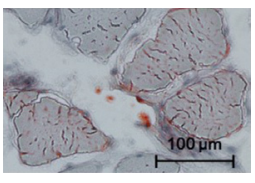

HFD

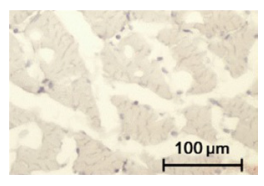

HFD+EXE

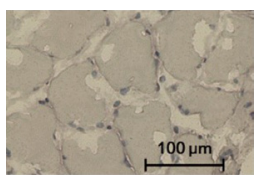

DA

A



B

Fig. 2. (A) The number of red Oil Red O-stained areas was significantly higher in the HFD group than in the other groups. (B) Greater intramyocellular lipids (IMCL) accumulation was observed in the soleus muscles of the HFD group. HFD, high-fat diet; HFD+EXE, combined high-fat diet and exercise; DA, dietary adjustment.

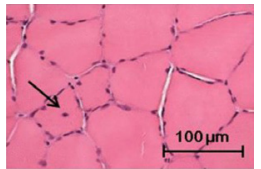

HFD

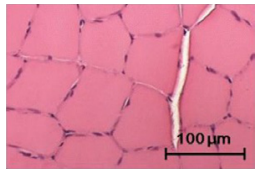

$H F D+E X E$



DA
A

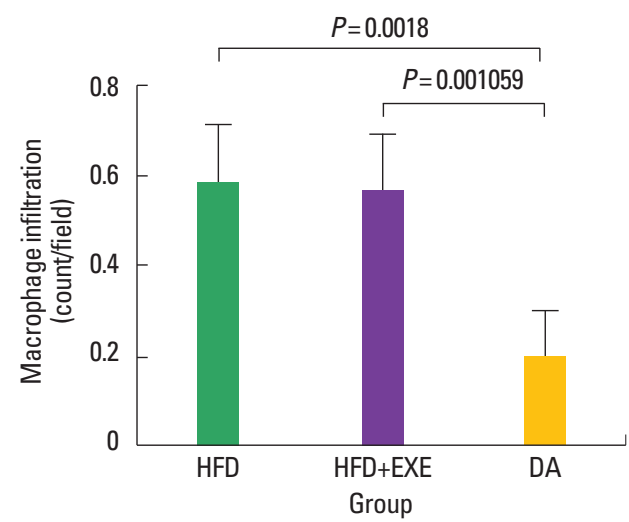

B

Fig. 3. (A) Exercise and high-fat diet induced lipid accumulation, which increased macrophage infiltration in the soleus muscle (arrow). (B) Macrophage infiltration was significantly $(P<0.001)$ increased in the high-fat diet-fed groups (HFD and HFD+EXE) compared to the DA group. HFD, high-fat diet; HF$\mathrm{D}+\mathrm{EXE}$, combined high-fat diet and exercise; DA, dietary adjustment.

\section{IMCL accumulation in the soleus muscle}

Soleus muscle IMCL content, as measured by ORO staining and electron microscopy (Fig. 2A), was significantly $(P<0.05)$

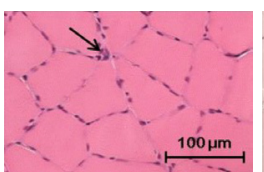

HFD

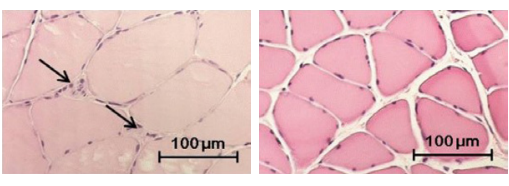

$\mathrm{HFD}+\mathrm{EXE}$
DA

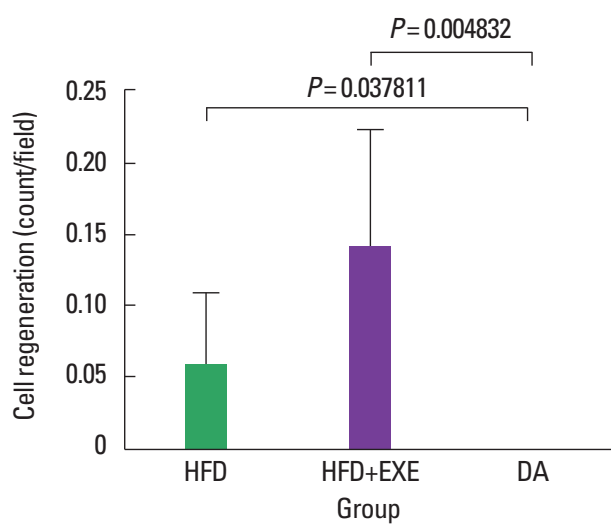

B

Fig. 4. (A) Exercise greatly stimulated cell regeneration (myogenesis) and the number of myogenic phenomena (arrows). (B) Cell regeneration was significantly higher in the HFD+EXE group compared to the HFD group and DA groups. HFD, high-fat diet; HFD+EXE, combined high-fat diet and exercise; DA, dietary adjustment.

higher in the HFD group compared with the HFD+EXE and DA groups (Fig. 2B).

\section{Macrophage infiltration and cell regeneration}

$\mathrm{H} \& \mathrm{E}$ staining results showed the degree of macrophage infiltration (Fig. 3) and cell regeneration (Fig. 4). Macrophage infiltration in the nucleus was significantly $(P<0.05)$ lower in the DA group than in the high-fat diet groups (HFD and HFD+EXE, Fig. 3B). Cell regeneration was significantly $(P<0.05)$ increased in the high-fat diet groups (HFD and HFD+EXE) compared to the DA group (Fig. 4B).

\section{Caspase-3 expression (activation level of apoptosis)}

Caspase-3 plays a pivotal role in apoptosis, we investigated whether its activity is increased in the soleus muscle with a high-fat diet or exercise. The expression of caspase- 3 was higher in the HFD+ EXE group than in the other group (Fig. 5A, B). There was no significant difference between HFD and DA groups. However, $\mathrm{HFD}+\mathrm{EXE}$ group was significant difference was larger than the $\operatorname{HFD}$ group $(P<0.001)$ when compared to the DA group $(P<0.05)$.

\section{DISCUSSION}

The present study provides evidence that exercise training in- 


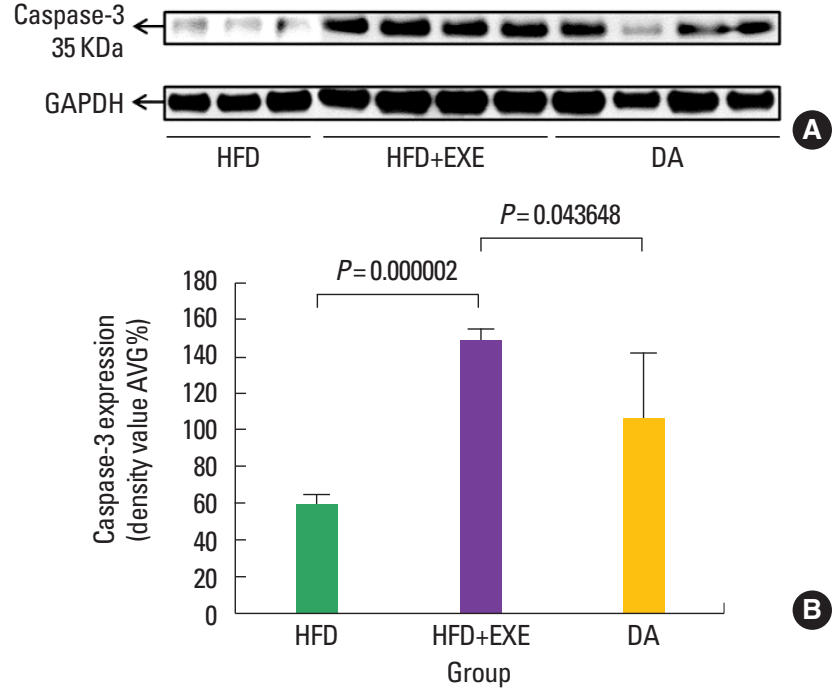

Fig. 5. High caspase-3 protein expression correlated with apoptosis. (A) Exercise stimulated apoptosis in the soleus muscle, indicating an increase in metabolism. (B) Caspase-3 expression was higher in the HFD+EXE group than the HFD group $(P<0.001)$ and the DA group $(P<0.05)$. HFD, high-fat diet; HFD+EXE, combined high-fat diet and exercise; DA, dietary adjustment.

fluences the level of IMCLs and cell survival in the soleus muscles of young adult rats. We showed that exercise training attenuates the extent of IMCL accumulation after 6 weeks of intervention. We demonstrated a positive relationship between the accumulation of IMCLs and macrophage infiltration and an inverse relationship between the accumulation of IMCLs and cell regeneration. We also found that exercise training is positively correlated with cell regeneration and caspase- 3 activity.

Our data showed that lipid accumulation in the cells was lower when the high-fat diet and the endurance exercise were prescribed simultaneously compared with the high-fat diet alone. These results are also consistent with the hypothesis that IMCL accumulation plays a role in lipid signaling, cellular damage, and maintenance of the homeostasis of muscle apoptotic factors. Moreover, reduced lipid accumulation as well as increased relative muscle weight in animals performing exercise might be due to increased cell regeneration. In light of this evidence, we suggest that exercise is more effective than dietary adjustment in ameliorating high-fat diet-induced lipid accumulation in the intramyocellular space.

Endurance training increases insulin sensitivity of skeletal muscle and increases lipid accumulation in muscles due to increased oxidative capacity (Goodpaster et al., 2001; Horton, 1986; Van Herpen and Schrauwen-Hinderling, 2008). However, in conjunction with a high-fat diet, insulin resistance and lipid accumula- tion in the skeletal muscle also increase (Mikines et al., 1988). Therefore, the increase in IMCL in subjects who partake in endurance training compared with normal subjects is due to increase in oxidative capacity and insulin sensitivity. However, in this study, the decrease in IMCL accumulation compared to animals consuming a high-fat diet is due to the different baseline fat amounts; even with a high-energy (high-fat) diet, regular, moderate exercise alone increased insulin sensitivity and decreased IMCL accumulation. This means that exercise can be effective at increasing insulin sensitivity in patients with T2DM, regardless of diet, and can also increase the oxidative capacity of skeletal muscle.

A previous study focused on lipid accumulation through exercise and diet. Specifically, Dubé and colleagues reported that the accumulation of IMCLs is increased by endurance exercise, and this phenomenon is called the "athlete's paradox" (Carraro and Franceschi, 1997). In a follow-up study, this phenomenon was shown to be even stronger in endurance-trained athletes (Dubé et al., 2011). However, these studies did not measure the phenomenon of IMCL accumulation in relation to a high-fat diet, either alone or when combined with exercise. Thus, that will not be known how do change lipid accumulation (Dubé et al., 2008; Goodpaster et al., 2001; Stannard and Johnson, 2004; van Loon and Goodpaster, 2006; Van Loon et al., 2004). Based on the ORO staining results in this study, exercise increased the oxidative capacity and glycogen content of soleus muscle cells. Additionally, exercise training helped reduce lipid accumulation in the cells. Furthermore, there was a significant difference in the relative muscle weight of the HFD+EXE group compared with the HFD group. Taken together, these results show the effects of exercise because the high-fat diet was given to both groups. Significant changes in the accumulation of IMCLs were not observed between the DA group and the HFD+EXE group. The HFD+EXE group showed higher relative muscle weight than the DA group, but the differences were not statistically significant due to large standard deviations.

The accumulation of IMCL was higher in the HFD group compared with the HFD+EXE group and the DA group. Therefore, macrophage infiltration and cell regeneration showed a positive correlation. Although there was no difference in the protein levels of caspase- 3 , the excessive lipid accumulation independently stimulated apoptosis. The HFD+EXE group showed high degrees of macrophage infiltration, inflammation, and cell regeneration, as well as elevated caspase- 3 protein concentration, but a low level of IMCL. This is consistent with previous results that showed that exercise can increase inflammation and apoptosis (Louis et al., 
2007; Siu et al., 2004). Finally, cell regeneration was stimulated through exercise, and the relative muscle weight was higher in the HFD+EXE group compared with the HFD group. As a result, excessive accumulation of IMCL and exercise independently stimulated cell damage. Exercise-related apoptosis and cell regeneration resulted in muscle growth. Excessive lipid accumulation, which is related to cell damage, resulted in a reduction in muscle; this is a negative stimulus.

Our results suggest that exercise is a more effective prescription than dietary adjustment for several metrics of muscle health. However, none of the parameters except cell damage-related factors showed significant changes between the groups. In addition, exercise has positive effects on skeletal muscles such as increased insulin sensitivity and fat-oxidation capacity (Ivy et al., 1986; Soman et al., 1979).

In conclusion, our data showed that both exercise and dietary adjustment had a positive effect on the reduction of lipid accumulation in muscle cells. Dietary adjustment was slightly more effective when viewed only as a reduction in lipid accumulation, but it showed that the dietary adjustment group exhibited significantly lower muscle weight and cell regeneration. Therefore, exercise is thought to be more effective than diet for treating cell damage due to lipid accumulation in muscle.

\section{CONFLICT OF INTEREST}

No potential conflict of interest relevant to this article was reported.

\section{ACKNOWLEDGMENTS}

This work was supported by the National Research foundation of Korea (NRF) grant funded by the Korea government (The Ministry of Education) (No. KRF-2009-332-G00071).

\section{REFERENCES}

Baek KW, Cha HJ, Park JJ. Effects of high-fat diet on type-I muscle loss in rats. J Life Sci 2013;23:1509-1515.

Bastie CC, Hajri T, Drover VA, Grimaldi PA, Abumrad NA. CD36 in myocytes channels fatty acids to a lipase-accessible triglyceride pool that is related to cell lipid and insulin responsiveness. Diabetes 2004;53: 2209-2216.

Boor P, Celec P, Behuliak M, Grancic P, Kebis A, Kukan M, Pronayová N, Liptaj T, Ostendorf T, Sebeková K. Regular moderate exercise reduces advanced glycation and ameliorates early diabetic nephropathy in obese Zucker rats. Metabolism 2009;58:1669-1677.

Borghouts LB, Keizer HA. Exercise and insulin sensitivity: a review. Int J Sports Med 2000;21:1-12.

Bradley RL, Jeon JY, Liu FF, Maratos-Flier E. Voluntary exercise improves insulin sensitivity and adipose tissue inflammation in diet-induced obese mice. Am J Physiol Endocrinol Metab 2008;295:E586-E594.

Carraro U, Franceschi C. Apoptosis of skeletal and cardiac muscles and physical exercise. Aging (Milano) 1997;9:19-34.

Devlin JT. Effects of exercise on insulin sensitivity in humans. Diabetes Care 1992;15:1690-1693.

Dubé JJ, Amati F, Stefanovic-Racic M, Toledo FG, Sauers SE, Goodpaster $\mathrm{BH}$. Exercise-induced alterations in intramyocellular lipids and insulin resistance: the athlete's paradox revisited. Am J Physiol Endocrinol Metab 2008;294:E882-E888.

Dubé JJ, Amati F, Toledo FG, Stefanovic-Racic M, Rossi A, Coen P, Goodpaster BH. Effects of weight loss and exercise on insulin resistance, and intramyocellular triacylglycerol, diacylglycerol and ceramide. Diabetologia 2011;54:1147-1156.

Dube JJ, Bhatt BA, Dedousis N, Bonen A, O'Doherty RM. Leptin, skeletal muscle lipids, and lipid-induced insulin resistance. Am J Physiol Regul Integr Comp Physiol 2007;293:R642-R650.

Goodpaster BH, He J, Watkins S, Kelley DE. Skeletal muscle lipid content and insulin resistance: evidence for a paradox in endurance-trained athletes. J Clin Endocrinol Metab 2001;86:5755-5761.

Goodyear LJ, Kahn BB. Exercise, glucose transport, and insulin sensitivity. Annu Rev Med 1998;49:235-261.

Hegarty BD, Cooney GJ, Kraegen EW, Furler SM. Increased efficiency of fatty acid uptake contributes to lipid accumulation in skeletal muscle of high fat-fed insulin-resistant rats. Diabetes 2002;51:1477-1484.

Horton ES. Exercise and physical training: effects on insulin sensitivity and glucose metabolism. Diabetes Metab Rev 1986;2:1-17.

Ivy JL, Sherman WM, Cutler CL, Katz AL. Exercise and diet reduce muscle insulin resistance in obese Zucker rat. Am J Physiol 1986 Sep;251(3 Pt 1):E299-E305.

Kusminski CM, Shetty S, Orci L, Unger RH, Scherer PE. Diabetes and apoptosis: lipotoxicity. Apoptosis 2009;14:1484-1495.

Louis E, Raue U, Yang Y, Jemiolo B, Trappe S. Time course of proteolytic, cytokine, and myostatin gene expression after acute exercise in human skeletal muscle. J Appl Physiol (1985) 2007;103:1744-1751.

Mikines KJ, Sonne B, Farrell PA, Tronier B, Galbo H. Effect of physical exercise on sensitivity and responsiveness to insulin in humans. Am J Physiol 1988;254(3 Pt 1):E248-E259.

Phaneuf S, Leeuwenburgh C. Apoptosis and exercise. Med Sci Sports Exerc 2001;33:393-396. 
Phillips DI, Caddy S, Ilic V, Fielding BA, Frayn KN, Borthwick AC, Taylor $\mathrm{R}$. Intramuscular triglyceride and muscle insulin sensitivity: evidence for a relationship in nondiabetic subjects. Metabolism 1996;45:947-950.

Schrauwen-Hinderling VB, Hesselink MK, Moonen-Kornips E, Schaart G, Kooi ME, Saris WH, Schrauwen P. Short-term training is accompanied by a down regulation of ACC2 mRNA in skeletal muscle. Int J Sports Med 2006;27:786-791.

Schrauwen-Hinderling VB, Kooi ME, Hesselink MK, Moonen-Kornips E, Schaart G, Mustard KJ, Hardie DG, Saris WH, Nicolay K, Schrauwen $\mathrm{P}$. Intramyocellular lipid content and molecular adaptations in response to a 1-week high-fat diet. Obes Res 2005;13:2088-2094.

Schrauwen P. High-fat diet, muscular lipotoxicity and insulin resistance. Proc Nutr Soc 2007;66:33-41.

Siu PM, Bryner RW, Martyn JK, Alway SE. Apoptotic adaptations from exercise training in skeletal and cardiac muscles. FASEB J 2004;18: 1150-1152.

Soman VR, Koivisto VA, Deibert D, Felig P, DeFronzo RA. Increased insulin sensitivity and insulin binding to monocytes after physical train- ing. N Engl J Med 1979;301:1200-1204.

Stannard SR, Johnson NA. Insulin resistance and elevated triglyceride in muscle: more important for survival than "thrifty" genes? J Physiol 2004;554(Pt 3):595-607.

Van Heek M, Compton DS, France CF, Tedesco RP, Fawzi AB, Graziano MP, Sybertz EJ, Strader CD, Davis HR Jr. Diet-induced obese mice develop peripheral, but not central, resistance to leptin. J Clin Invest 1997;99:385-390.

van Herpen NA, Schrauwen-Hinderling VB. Lipid accumulation in non-adipose tissue and lipotoxicity. Physiol Behav 2008;94:231-241.

van Loon LJ, Goodpaster BH. Increased intramuscular lipid storage in the insulin-resistant and endurance-trained state. Pflugers Arch 2006;451: 606-616.

van Loon LJ, Koopman R, Manders R, van der Weegen W, van Kranenburg GP, Keizer HA. Intramyocellular lipid content in type 2 diabetes patients compared with overweight sedentary men and highly trained endurance athletes. Am J Physiol Endocrinol Metab 2004;287: E558-E565. 